\title{
Research on Intelligent Predictive Analysis System Based on Embedded Wireless Communication Network
}

\author{
Jingwei Sun \\ Hefei University of Technology, Hefei 230001, China \\ Correspondence should be addressed to Jingwei Sun; 2014010059@mail.hfut.edu.cn
}

Received 9 December 2021; Revised 18 January 2022; Accepted 9 February 2022; Published 27 February 2022

Academic Editor: Mu Zhou

Copyright ( 2022 Jingwei Sun. This is an open access article distributed under the Creative Commons Attribution License, which permits unrestricted use, distribution, and reproduction in any medium, provided the original work is properly cited.

\begin{abstract}
In order to improve the practical effect of intelligent prediction system, by analyzing and studying the characteristics of the traffic carried on the network, it can provide an effective way to explore the internal operating mechanism of the network. This paper takes the embedded wireless communication network as the research object to construct an intelligent predictive analysis system and applies the intelligent regression algorithm to the intelligent predictive analysis to construct an intelligent predictive analysis system. Finally, this paper verifies the system model of this paper through experimental research. The research results show that the intelligent predictive analysis system based on the embedded wireless communication network proposed in this paper is very effective and has a positive effect on the construction and development of the embedded wireless communication network.
\end{abstract}

\section{Introduction}

Wireless local area network is the product of the combination of computer network and wireless communication technology. Specifically, traditional cables are no longer used when setting up a local area network but are connected wirelessly using infrared rays, radio waves, etc. as the transmission medium to provide all the functions of a wired local area network [1]. The basis of the wireless local area network is the traditional wired local area network, which is the expansion and replacement of the wired local area network. It realizes wireless communication through wireless hubs, wireless access nodes, wireless network bridges, wireless network cards, and other devices on the basis of the wired local area network. Currently, the frequency band used by wireless local area networks is mainly S-band $(2.4 \mathrm{GHz}-$ $2.4835 \mathrm{GHz}$ ). The networking mode of wireless local area network can be roughly divided into two types, one is adhoc mode, that is, point-to-point wireless network, and the other is infrastructure mode, that is, centralized control network [2]. The wireless local area network can make up for the deficiencies of wired Ethernet that rely on cables or optical cables in some special application environments and realize the extension of the network. Embedded system integration of wireless local area network technology to achieve wireless communication and data transmission will become a hot spot for future applications. For example, wireless digital set-top boxes, computers, wireless gateways, and household appliances can form a home wireless local area network, and at the same time can be connected to the Internet through an AP, a wireless router, or a wireless bridge. The wireless instrument performs data collection and wireless transmission. Wireless instruments and equipment are arranged in the work site with an ad hoc network for mutual information transmission and remote wireless monitoring, which reduces the trouble and inconvenience of wiring, greatly improves industrial production efficiency and facilitates people's lives [3].

With the further development of embedded operating systems and wireless communication technologies, data terminals relying on wireless network data transmission based on embedded operating systems have become more and more widely used. Embedded operating system is a hot research topic nowadays, and embedded Linux is stable, efficient, easy to customize, easy to cut, extensive hardware support, free, open source, and other characteristics, which 
makes Linux widely used in the embedded field. In recent years, the development of global communication technology has been changing with each passing day. Especially in the past two to three years, the development speed and application fields of wireless communication technology have surpassed that of fixed communication technology, showing a trend of development in full swing. The most representative ones are cellular mobile communications, broadband wireless access, as well as trunking communications, satellite communications, and mobile video services and technologies.

Due to the limitation of limited communication and sampling period, the current distributed prediction methods for multiagent systems are mostly noniterative and cooperative algorithms. In each sampling period, there is only one information exchange between the agents, and only local performance indicators are calculated.

This paper studies the application of intelligent network and the behavior characteristics of the network itself and obtains parameter information through the study of network traffic. By analyzing and studying the traffic characteristics carried on the network, combined with the embedded wireless communication network, the intelligent prediction and analysis system is constructed, the intelligent prediction and regression algorithm is improved, the operation effect of the intelligent prediction and analysis system is improved, and the practical effect of the intelligent prediction system is improved.

This article takes the embedded wireless communication network as the research object to construct the intelligent predictive analysis system and verify and analyze its performance, which provides a theoretical reference for the further development of the subsequent wireless communication network technology.

\section{Related Work}

The modeling research on networked predictive control is as follows:

Literature [4] proposed the concept of networked predictive control, which actively compensates for the communication delay and data packet loss in the control loop through iterative prediction. In this paper, a networked predictive control strategy is designed for a class of linear timeinvariant systems to solve the steady time delay and the random communication time delay, respectively. Literature [5] studied the problem of networked predictive control in the presence of random network delays in the feedback channel for a type of multiple-input multiple-output discrete-time general system, carried out numerical simulation research, and based on the NetCon-ARM9 embedded system platform. Verify the effectiveness of the above control strategy. For a type of linear system described by a discrete-time model, some scholars have studied the networked predictive control problem in the case of communication delays in both the forward channel and the feedback channel [6]. Literature [7] gives the necessary and sufficient conditions to ensure the stability of the closed-loop system when the communication delays are constant. At the same time, when the random delay in the communication channel is bounded, if the corresponding switching system is stable, then the obtained closed-loop networked predictive control system is also stable. Simulations and experiments have verified the above conclusions. Literature [8] considered the predictive control problem in the case of disturbances in both the forward channel and the feedback channel of the system model. Literature [9] established a model based on the Markov chain for the communication delay in the two-way channel, using a local Lomberg observer to estimate the state vector that could not be measured, and the measurement output and the estimated state vector through the limited communication capacity. The communication channel is sent to the tracking controller, which solves the problem of networked predictive tracking control. When the random communication delay exists in the control loop of the networked control system, literature [10] proposed an output feedback predictive controller to actively compensate for the communication delay and proved the stability of the closed-loop networked predictive control system.

The research on network predictive control method and data processing is as follows:

In the above research results of the networked predictive control method, the controller is generally required to calculate the control value at each time in the future and then package it and send it to the controlled object via the communication network to actively compensate for various possible time delays. At the end of the controlled object, the most appropriate control value is selected based on the measured delay size, so as to realize the delay compensation in the forward channel [11]. However, this method needs to know the accurate mathematical model of the controlled object in advance and needs to measure the accurate communication time lag, which is difficult to achieve for most applications. Literature [11] proposed a data-driven networked predictive control method for a type of single-input singleoutput discrete-time autoregressive moving average model. This method does not need to accurately measure the delay in the communication network, and allows the existence of uncertain parameters of the system model.

The related research on the construction of intelligent prediction system for wireless communication network is as follows:

In the past, the research on traffic forecasting used a single model to describe the original characteristics of traffic. However, there are more and more uncertain factors affecting network traffic characteristics (such as new applications and protocol structure changes). When a single model is used for forecasting, large errors will inevitably occur [12]. Therefore, a single prediction model, such as Markov model, ARIMA model, and mock-MA model, cannot predict network traffic well because it only describes some of the characteristics of traffic. Although a single neural network model and support vector machine model can describe the characteristics of network traffic more comprehensively, their prediction accuracy for complex network traffic is still not satisfactory. According to the fact that there are multiple characteristics of network traffic, many scholars use different models to describe the corresponding characteristics and then combine them to predict network traffic and achieve better prediction results than a single model. Literature 
[13] has no extraction in Haar. On the basis of wavelet transform, combined with adaptive AR model and sliding window polynomial fitting method, a recursive high-speed network traffic online prediction model based on wavelet transform is established. This model not only improves the accuracy of online traffic prediction but also avoids regular estimation and update of parameters through the recursive automatic adjustment of model parameters. The literature [14] combines wavelet analysis and Kalman filtering and uses Kalman filtering to deal with the linear change part of network traffic, use wavelet analysis to deal with the nonlinear change part, and simulation results show that the model has high prediction accuracy; literature [15] uses the FARtMA model to describe the long correlation and short correlation, and the neural network to describe the nonstationary. Finally, the results of the two models are optimized and combined; literature [16] combines wavelet analysis and neural network to establish a prediction model. First, wavelet decomposition is used to decompose the network traffic data into wavelet coefficients and scale coefficients and the coefficients of these different frequency components. A single branch is reconstructed into high-frequency components and low-frequency components. The FIR neural network is used to predict these components separately, and the synthesized result is used as the prediction of the original network traffic, which has achieved good results; literature [17] uses the wavelet method to predict. The network traffic is preprocessed, and then the linear neural network and the Elman neural network are used to make predictions, respectively, to ensure that the correlation and nonstationarity of the traffic can be described. Finally, the two prediction results are synthesized into the final prediction result through the BP neural network. It shows that the combined model has higher prediction accuracy than the single model.

Literature [18] proposes a novel heuristic to reconstruct application-layer messages in the common case of encrypted traffic. We discuss and experimentally evaluate the suitability of the provided modeling approaches for different tasks.

Literature [19] investigates and specializes a set of architectures selected among convolutional, recurrent, and composite neural networks, to predict mobile-app traffic at the finest (packet-level) granularity.

In order to overcome the problems existing in the intelligent predictive analysis system, this paper combines the embedded wireless communication network to improve the effect of the intelligent predictive analysis system. The first part describes the current situation and background and summarizes the existing problems and the research content of this paper; the second part is the literature review part, which analyzes the research of experts and scholars on related issues. Therefore, the organizational structure of this paper, the third part is the algorithm improvement part, which applies the intelligent regression algorithm to the intelligent prediction analysis, and builds an intelligent prediction analysis system; in the fourth part, the intelligent predictive analysis system is improved and constructed, and the effect of the system is verified by experiments. Finally, the research content of this paper is summarized and prospected.

\section{Intelligent Predictive Regression Algorithm}

We set the regression function as $m(x) \in C[a, b]$ and assume that $\left\{\varphi_{i}\right\}_{i=0}^{\infty}$ constitutes a set of orthogonal basis on $[a, b]$, namely,

$$
\int_{a}^{b} \varphi_{i}(x) \varphi_{j}(x) d x=\delta_{i j}=\left\{\begin{array}{l}
0, i \neq j, \\
c_{i}, i=j .
\end{array}\right.
$$

Because of $\int \prod_{i=1}^{d} \varphi_{i j}\left(x_{i}\right) \prod_{i=1}^{d} \varphi_{i k}\left(x_{i}\right) d x_{1} \cdots d x_{d}=\prod_{i=1}^{d} \int$ $\varphi_{i j}\left(x_{i}\right) \varphi_{i k}\left(x_{i}\right) d x_{i}=\prod_{i=1}^{d} \delta_{j k}=\delta_{j k},\left\{\prod_{i=1}^{d} \varphi_{i j}\left(x_{i}\right)\right\}_{i=1}^{\infty}$ constitutes a set of orthogonal bases on $\prod_{i=1}^{d}\left[a_{i}, b_{i}\right]$, then $m(x)$ has an orthogonal sequence expansion $m(x)=\sum_{i=1}^{\infty} \theta_{i} \prod_{k=1}^{d} \varphi_{k i}(x)$. Therefore, the nonparametric regression model can be approximated as

$$
Y_{i}=\sum_{j=1}^{m} \theta_{i} \prod_{k=1}^{d} \varphi_{k i}\left(x_{k i}\right)+v_{i}
$$

By performing least squares estimation on the model, we get

$$
\widehat{\theta}=\left(Z^{T} Z\right)^{-1} Z^{T} Y
$$

Among them, $\quad Z=\left(Z_{1}, \cdots, Z_{m}\right), Z_{i}=$ $\left(\prod_{j=1}^{d} \varphi_{j i}\left(X_{i 1}\right), \cdots, \prod_{j=1}^{d} \varphi_{j i}\left(X_{\text {in }}\right)\right)^{T}$.

Then, $m(x)$ has an orthogonal sequence estimate [20]:

$$
\widehat{m}_{n}(x)=z(x)^{T} \widehat{\theta} .
$$

Among them, $\left.z(x)=\prod_{i=1}^{d} \varphi_{i 1}\left(x_{i}\right), \cdots, \prod_{i=1}^{d} \varphi_{i m}\left(x_{i}\right)\right)^{T}$.

We set $v(x)=\sigma_{n}^{2}\left(z(x)^{T}\left(Z^{T} Z\right)^{-1} z(x)\right)$. When $n \longrightarrow \infty$, $m \longrightarrow \infty$, the orthogonal sequence estimation has the following properties:

(1) $v(x)^{-1 / 2}\left(\widehat{m}_{n}(x)-E \widehat{m}_{n}(x)\right) \underline{d} N(0,1)$

(2) $v(x)^{-1 / 2}\left(E \widehat{m}_{n}(x)-m\right) \longrightarrow 0$

(3) $\sigma_{n}^{2}=n^{-1} \sum_{i=1}^{n}\left(Y_{i}-\widehat{m}_{n}\left(X_{i}\right)\right)^{2}$ is a consistent estimate of $\sigma_{n}^{2}$

In orthogonal sequence estimation, the Legendre polynomial orthogonal basis is often selected, and its representation is as follows:

$$
\begin{gathered}
P_{0}(x)=\frac{1}{\sqrt{2}}, \\
P_{1}(x)=\frac{x}{\sqrt{2 / 3}} .
\end{gathered}
$$

Other high-order Legendre polynomials can be recursively obtained by the following formula: 


$$
(m+1) P_{m+1}(x)=(2 m+1) x P_{m}(x)-m P_{m-1}(x) .
$$

The orthogonal basis $\left\{P_{i}(x)\right\}_{i=0}^{\infty}$ of Legendre polynomial satisfies

$$
\int_{1} P_{i}(x) P_{j}(x) d x=\delta_{i j}=\left\{\begin{array}{l}
0, i \neq j \\
1, i=j .
\end{array}\right.
$$

If the independent variable $X$ takes a value in the interval $[a, b]$, the variable $Z=2 X-a-b / b-a$ must be replaced so that the value interval of the variable $Z$ is $[-1,1]$.

Orthogonal sequence estimation will also select Fourier orthogonal basis, which is defined as follows:

$$
\begin{gathered}
q_{1}(x)=1, \quad q_{2 k}(x)=\sqrt{2} \cos (2 \pi k x), \\
q_{2 k+1}(x)=\sqrt{2} \sin (2 \pi k x)(k=1,2, \cdots) .
\end{gathered}
$$

Fourier orthogonal basis $\left\{q_{i}(x)\right\}_{i=1}^{\infty}$ satisfies

$$
\int_{b}^{1} q_{i}(x) q_{j}(x) d x=\left\{\begin{array}{l}
0, i \neq j \\
1, i=j .
\end{array}\right.
$$

Similarly, if the independent variable $x$ takes a value in the interval $[a, b]$, the variable must be replaced with $Z=X-a / b-a$ so that the value interval of the variable $Z$ is $[0,1][21]$.

In practical problems, the linear relationship between variables will in most cases change with another covariate (such as time, temperature, etc.). Since the data information when the next covariate changes are unknown, a question arises: how to use the known historical data to estimate the parameters of the linear model when the next covariate changes.

Regarding the parameter estimation of the variable coefficient regression model, the local weighted least squares method can be used to introduce the concept of variable coefficient weighted estimable function to construct parameter estimators. On the basis of the local weighted least squares method, this paper combines the relevant content in nonparametric regression to estimate the variable coefficients by weighted least squares.

Nonparametric regression models can be divided into two categories, complete nonparametric regression models (referred to as non-parametric regression models) and semiparametric regression models. The variable coefficient regression model is a special case in the semiparametric regression model.

We assume that the independent variable $x_{1}, x_{2}, \cdots, x_{p}$ and the dependent variable $y$ satisfy a linear relationship at the parameter $t$.

$$
y=\beta_{0}(t)+\beta_{1}(t) x_{1}+\cdots \beta_{p}(t) x_{p}
$$

Among them, $\beta_{i}(t), i=0,1,2, \cdots, p$ is a bounded continuous function of a one-dimensional (or multidimensional) real variable $b$ and has a continuous derivative, and $\beta_{i}(t)$ is called a variable coefficient.

We assume that $t_{1}, t_{2}, t_{3}, \cdots, t_{n}$ is $n$ points of $t_{0}$ near a specified point, and the sample observation value $\left(y_{i}, t_{i}, x_{i}\right.$, $\left.\cdots, x_{i p}\right)$ is obtained by observing at each point.

$$
y_{i}=\beta_{0}\left(t_{i}\right)+\beta_{1}\left(t_{i}\right) x_{i 1}+\cdots \beta_{p}\left(t_{i}\right) x_{i p}+\varepsilon_{i}
$$

Among them, $\varepsilon_{1}, \varepsilon_{2}, \cdots, \varepsilon_{n}, E \varepsilon_{i}=0, \operatorname{Var}_{i}=\sigma^{2}, i=1,2$, $\cdots, n$.

The variable coefficient regression model uses the observation value $\left(y_{i}, t_{i}, x_{i 1}, \cdots, x_{i p}\right)$ at the point $t_{i}$ near $t_{0}$ to estimate the parameter $\beta_{i}(t)$ at $t_{0}$. Since the observations at different $t_{i}$ have different "importance" relative to $t_{0}$, the weight function $w_{i}\left(t_{o}\right)$ needs to be used to measure.

This paper uses the distance between the observation value at $t_{i}$ and $t_{0}$ to define the relationship between them, namely,

$$
w_{i}\left(t_{o}\right)=w\left(p\left(v_{i}, v_{o}\right)\right)
$$

Among them, $p\left(v_{i}, v_{o}\right)$ represents the Euclidean distance from $v_{i}$ to $v_{o}, i=1,2, \cdots, n$.

The most commonly used weight function estimation is kernel estimation and nearest neighbor estimation.

The probability density function $K(\cdot)$ with symmetric origin is selected:

$$
\int K(u) d u=1
$$

is the kernel function and window width $h_{n}>0$. The nuclear weight function is defined as

$$
W_{n i}(x)=\frac{K_{h_{n}}\left(X_{i}-x\right)}{\sum_{j=1}^{n} K_{h_{2}}\left(X_{j}-x\right)} .
$$

Among them, $K_{h_{n}}(u)=h_{n}^{-1} K\left(u h_{n}^{-1}\right)$ is also a probability density function, and the parameter $h_{n}$ is called the window width.

The Nadaraya-Watson kernel estimate is defined as

$$
\widehat{m}_{n}(x)=\sum_{i=1}^{n} W_{n i}(x) Y_{i}
$$

The $K$-nearest neighbor non-parametric estimation algorithm is mainly composed of four parts: database generation, near neighbor sample subset search, near neighbor subset optimization, and forecast quantity estimation. The data flow relationship can be seen in Figure 1.

Four steps of the $K$-Nearest Neighbor Nonparametric Regression Prediction Algorithm.

(1) The more historical data, the more conducive the nonparametric regression estimation is to more truly and completely express the characteristics of the system state, and the more conducive to obtaining 


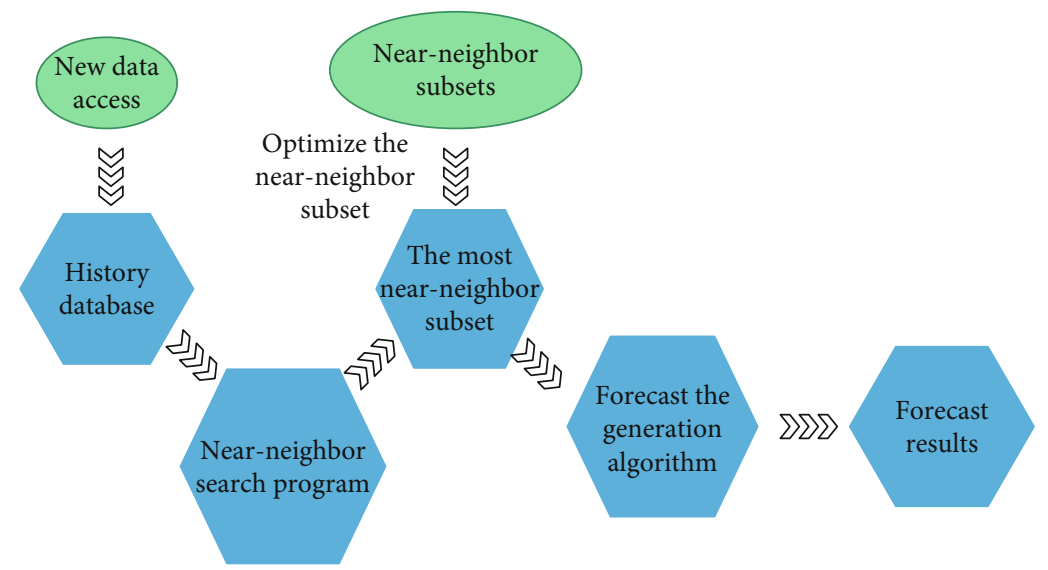

FIGURE 1: Schematic diagram of $K$-nearest neighbor nonparametric estimation algorithm.

accurate forecast estimates. Moreover, the historical database should be dynamic. As long as there is new data, it will be put into the database and the database data will be updated continuously

(2) The process of searching for neighbors is to find historical records similar to the current state condition characteristics in the historical database according to the predefined similarity measure and mark the searched historical records with similar characteristics as a neighbor. All the searched neighbors form a subset of neighbors

Step 1. Put the observed historical data into the database to build a complete historical database.

Step 2. Define a state vector that meets the requirements.

Step 3. Select the appropriate distance measurement method to determine the nearest neighbor search rules.

Step 4. Determine the appropriate $K$ value and select $K$ nearest neighbors in the historical database.

Step 5. Input the $K$-nearest neighbors determined in Step 4 into the prediction algorithm to obtain the predicted value.

The optimal neighbor subset refers to the subset formed by the neighbors that contribute the most to the forecast estimate among all the neighbors obtained by the search. Generally, it is determined by control parameters and optimization indexes. The control parameter of the $K$-nearest neighbor nonparametric estimation is the sample size of the optimal nearest neighbor subset. The optimization index can be similar to the index in the parameter estimation model. At present, the minimum prediction error sum of squares criterion is frequently used.

(3) After the optimal nearest neighbor subset is determined, the optimal subset can be used to estimate the production forecast. The state feature vector has been divided into condition feature vector and forecast feature vector in the database. In this way, each neighbor in the optimized neighbor subset has a corresponding predictor feature vector, and these predictor feature vectors can be regarded as the output corresponding to the conditional feature vector of each neighbor. The latest input conditional feature vector has a certain "distance" from each neighbor in the nearest neighbor subset. Therefore, it is necessary to synthesize the output vectors in all the best nearest neighbor subsets to get the most likely output vector corresponding to the conditional feature vector to be predicted. It is generally assumed that such a comprehensive operator is a linear operator or a random operator, and it can also be assumed to be a nonlinear operator. The most commonly used is the arithmetic average operator or the weighted average operator

The core problem of the $K$-nearest neighbor nonparametric estimation algorithm is the determination of the weight function of the nearest neighbor subset. By referring to the method of determining the weight function in the kernel weight estimation, this paper gives the following $K$ -nearest neighbor kernel weight estimation model.

We set $1<k<n$, and $J_{x, k}=\left\{i: X_{i}\right.$ is one of the $k$ nearest predicted values to $x\}$. Moreover, we combine the relevant theories in the kernel weight estimation to obtain the $K$ -nearest neighbor kernel weight estimate of the nonparametric regression model as

$$
\widehat{m}_{n}(x, k)=\frac{\sum_{i=1}^{n} K\left(\left(X_{i}-x\right) / R(x, k)\right) Y_{i}}{\sum_{i=1}^{n} K\left(\left(X_{i}-x\right) / R(x, k)\right)} .
$$

Among them, the function $K(\cdot)$ is the kernel function in the kernel weight estimation, and the similarity measure between the data is defined by the Euclidean distance, namely,

$$
R(x)=\max \left\{\left[(z-x)^{T}(z-x)\right]^{1 / 2}: z \in J_{x}\right\} .
$$


From equation (1), it can be seen that the $K$-nearest neighbor kernel weight estimation is the weighted average of the $k$ observations closest to $x$.

The commonly used kernel function for $K$-nearest neighbor kernel weight estimation is $K(u)=d(d+2) / 2 S_{d}$ $\left(1-u_{1}^{2}-\cdots-u_{d}^{2}\right)_{+}$, where $S_{d}=2 \pi^{d / 2} / \Gamma(d / 2)$. The $K$-nearest neighbor nuclear weight using a one-element kernel is estimated as

$$
\widehat{m}_{n}(x)=\frac{\sum_{i=1}^{n} K\left(R(x)^{-1}\left[\left(X_{i}-x\right)^{T}\left(X_{i}-x\right)\right]^{1 / 2}\right) Y_{i}}{\sum_{i=1}^{n} K\left(R(x)^{-1}\left[\left(X_{i}-x\right)^{T}\left(X_{i}-x\right)\right]^{1 / 2}\right)}
$$

The commonly used kernel functions are triangular kernel function $(1-|u|)_{+}$, parabolic kernel function 0.75 $\left(1-u^{2}\right)_{+}$, fourth power kernel function $15 / 16\left(\left(1-|u|^{2}\right)_{+}\right)^{2}$, and sixth power kernel function $70 / 81\left(\left(1-|u|^{3}\right)_{+}\right)^{2}$.

(i) In addition to retaining the characteristics of nuclear power estimation, the nearest neighbor nuclear power estimation also has properties such as consistency and asymptotic normality under appropriate conditions. The convergence speed at the interior point can reach $O\left(n^{-2 k d+4}\right)$

From this, the observation value at $t_{i}$ can be estimated to the k-nearest neighbor kernel weight at $t_{o}$ as

$$
W_{i}\left(t_{0}\right)=W\left(\rho\left(v_{i}, v_{0}\right)\right)=\frac{K\left(R(v)^{-1} \rho\left(v_{i}, v_{0}\right)\right)}{\sum_{i=1}^{n} K\left(R(v)^{-1} \rho\left(v_{i}, v_{0}\right)\right)}
$$

Among them, $R(v)=\max \left\{\rho\left(v_{i}, v_{0}\right): v_{i} \in J_{v}\right\}$.

According to regression estimation theory, when the error term of the established regression model has heteroscedasticity, the parameter estimation obtained by the ordinary least squares regression method is biased.

$\widehat{\beta}\left(t_{0}\right)$ is the weighted least squares estimate of $\beta\left(t_{0}\right)$. If $\widehat{\beta}\left(t_{0}\right)$ satisfies

$$
Q\left(\widehat{\beta}\left(t_{0}\right)\right)=\min _{\beta\left(t_{0}\right)} Q\left(\beta\left(t_{0}\right)\right)
$$

Among them,

$$
\begin{aligned}
\left(\beta\left(t_{0}\right)\right) & =\sum_{i=1}^{n} W_{i}\left(t_{0}\right)\left[y_{i}-\beta_{0}\left(t_{i}\right)-\beta_{1}\left(t_{i}\right) x_{i 1}-\cdots-\beta_{p}\left(t_{i}\right) x_{i p}\right]^{2} \\
& =\left(Y-X \beta\left(t_{0}\right)\right)^{T} W\left(t_{0}\right)\left(Y-X \beta\left(t_{0}\right)\right) .
\end{aligned}
$$

From

$$
\frac{\partial Q\left(\beta\left(t_{0}\right)\right)}{\partial \beta\left(t_{0}\right)}=2 X^{T} W\left(t_{0}\right) Y-2 X^{r} W\left(t_{0}\right) X \beta\left(t_{0}\right)=0 .
$$

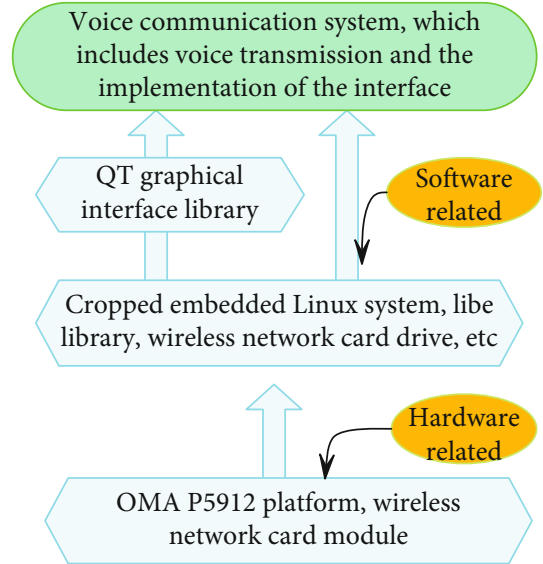

Figure 2: The overall framework of the system.

We obtain

$$
X^{T} W\left(t_{0}\right) X \beta\left(t_{0}\right)=X^{T} W\left(t_{0}\right) Y
$$

When $X^{T} W\left(t_{0}\right) X$ is reversible, we obtain

$$
\widehat{\beta}\left(t_{0}\right)=\left(X^{T} W\left(t_{0}\right) X\right)^{-1} X^{T} W\left(t_{0}\right) Y .
$$

\section{Intelligent Predictive Analysis System Based on Embedded Wireless Communication Network}

The wireless voice communication system implemented in this paper uses an embedded design model. After refinement, the entire system must complete the design of the following modules. The overall system framework is shown in Figure 2.

The overall design process of the system is divided into a system migration part, a software development part, and a graphical interface development part. The system migration part is to build an environment for the software part, including transplanting the Linux kernel and so on. The software part is to complete the voice transmission and reception, voice conversation management functions, and so on. The overall design process of the system is shown in Figure 3 below.

In order to assist the upper layer protocol to select a better link for data transmission and effectively improve the transmission efficiency of the network, real-time and accurate link quality prediction is required. Existing research shows that although PRR is the most common and direct link quality indicator for link quality evaluation, the PRR in a small-time window cannot accurately reflect the link quality, and long-term statistics are needed to obtain a more accurate PRR estimate. Therefore, the agility of directly using PRR for link quality prediction is usually very poor. Existing link quality prediction methods usually predict physical layer parameters such as RSSI, LQI, and SNR, and then evaluate the link quality based on the mapping model between the corresponding physical layer parameters and 


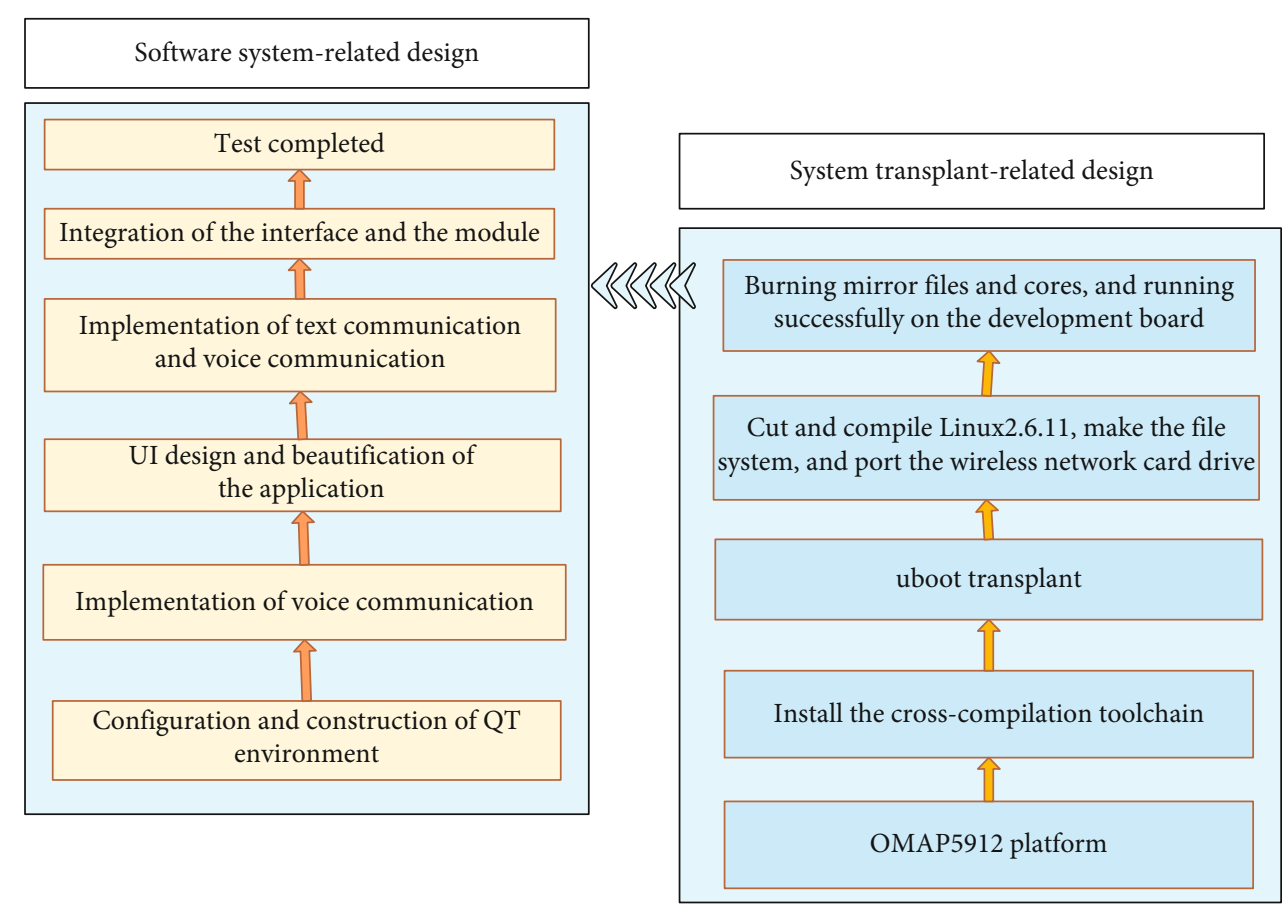

Figure 3: Flow chart of overall system design.

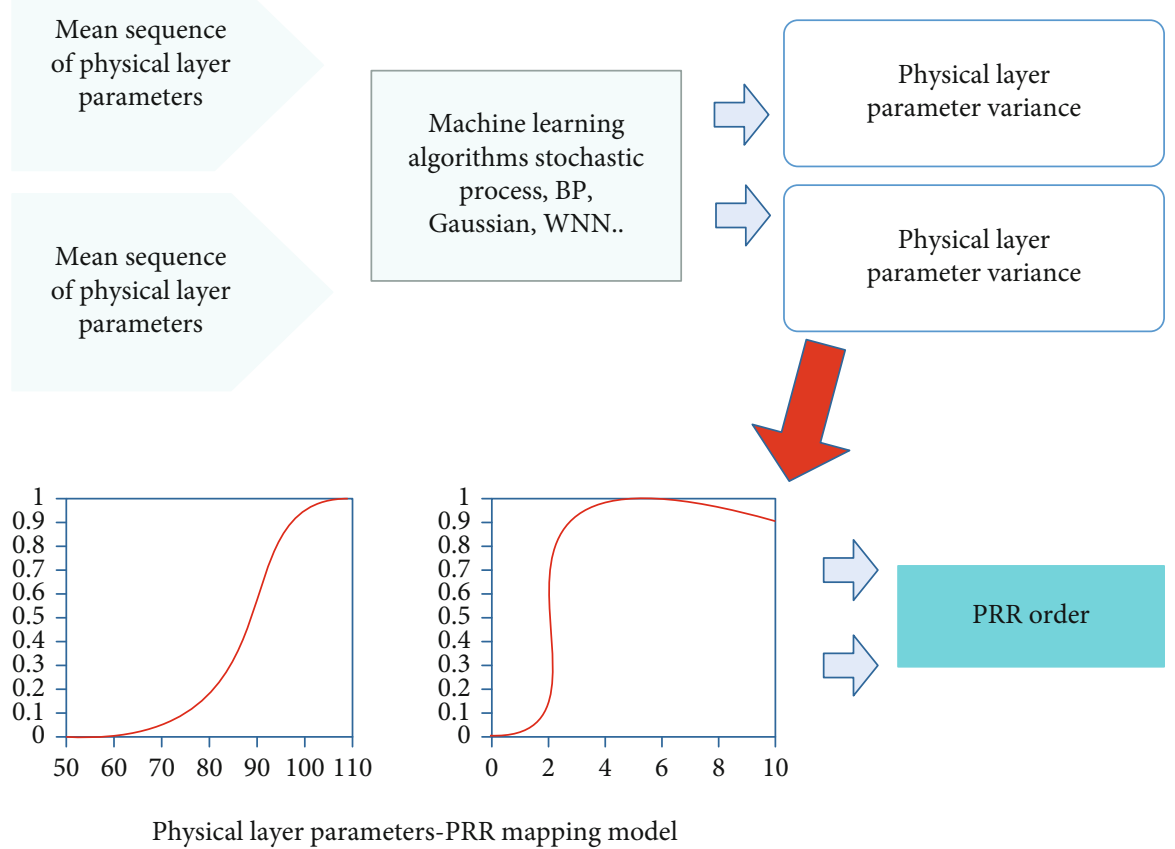

FIgURE 4: Traditional link quality prediction method model.

PRR. This can effectively solve the problem of poor agility in directly using PRR. Figure 4 summarizes the traditional wireless link quality prediction model.

The RNN-LQP proposed in this paper is shown in Figure 5. The predictor uses a recurrent neural network to predict the LQI counted in a small time window to ensure sufficient agility. Considering that the cyclic neural network has short-term memory characteristics and high prediction accuracy, it can also ensure sufficient accuracy and reliability. In addition, the predictor also selects LQI as the physical layer parameter.

In order to prevent data conflicts and network congestion and cause a large number of data packet loss, which will cause a serious negative impact on the performance of the 


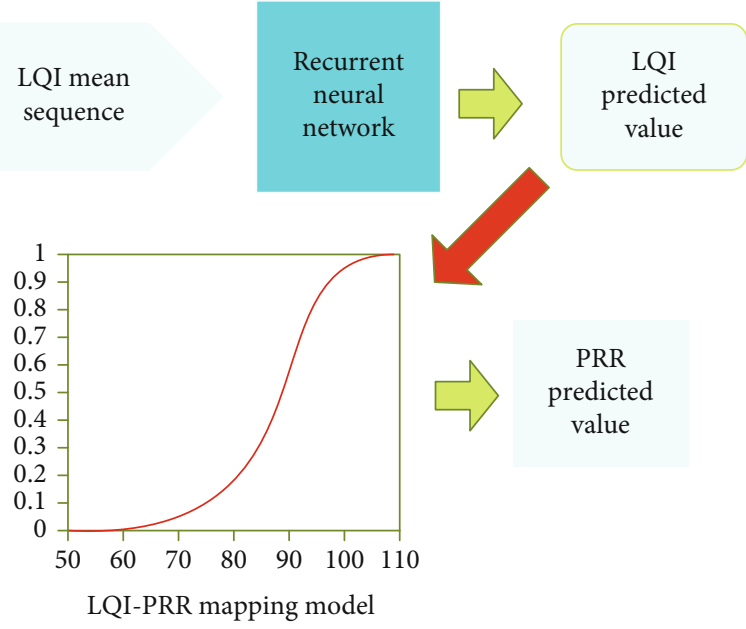

FigURE 5: RNN-LQP structure diagram.

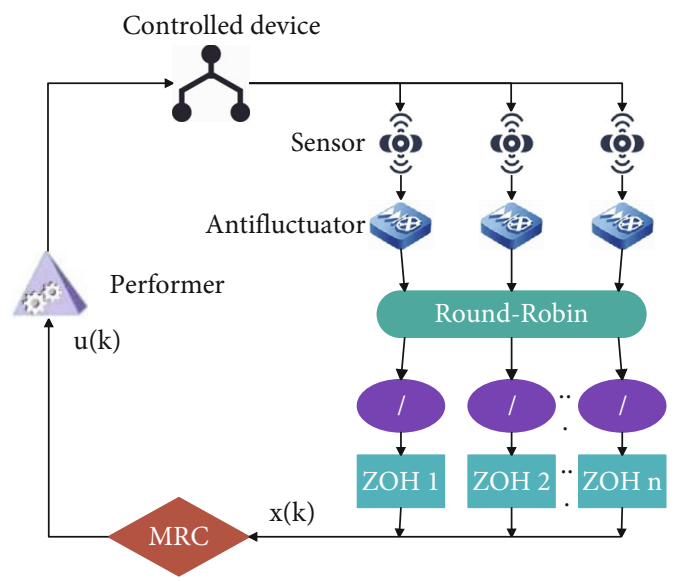

FIgURE 6: The structure diagram of the closed-loop system under the RR protocol.

system, this paper will use the RR protocol to schedule the data transmission sequence from the sensor to the controller. The structure of the control system based on the RR protocol is shown in Figure 6.

According to Figure 7, it can be obtained that both of the two robust MPC strategies proposed based on the cyclic communication protocol can make the closed-loop system finally reach an asymptotically stable state. In addition, according to Figure 8 , it can be known that the attractive domain of the robust MPC strategy with a free control function is larger than that of the robust MPC strategy without free control function. Therefore, appropriately increasing the free control function can make the optimization of the system more flexible and easier to achieve stability.

The big data prediction model can effectively extract the characteristics of the time series of the core performance indicators of the communication network, but it still has the defect of low data processing rate. In order to solve this problem, this paper models separately according to the classification results, reduces the model data dimension, and

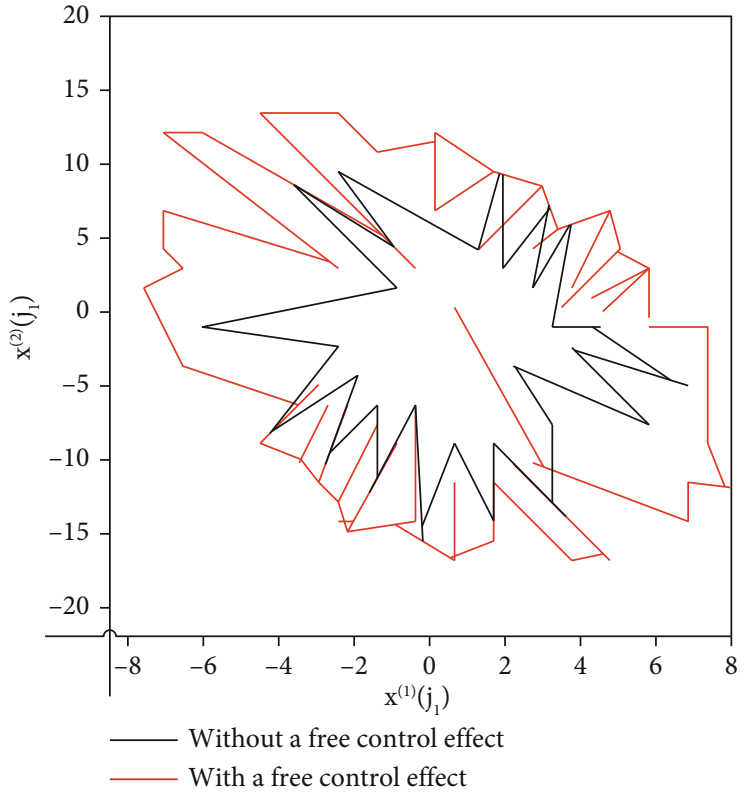

FIGURE 7: State trajectory of robust MPC based on RR protocol.

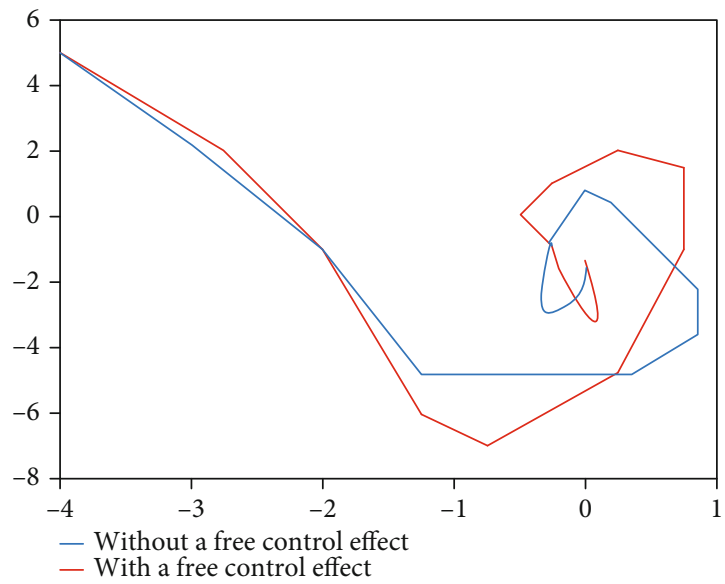

FIGURE 8: The attractive domain of robust MPC based on RR protocol.

improves the prediction rate. Figure 9 shows the flow chart of the improved big data prediction model based on support to the machine and association rules.

Although the analysis and prediction of massive data can improve the accuracy and reliability of the prediction results, the processing of massive data will inevitably bring defects such as slow prediction rate, so appropriate measures must be taken to increase the prediction rate. It is necessary to analyze the change law of the core performance indicators of the mobile communication network, use the change law to extract and classify the core performance indicators, and establish prediction models for different data segments of the performance indicators. On the one hand, data dimensions can be reduced, data processing time can be reduced, and the prediction rate can be improved. On the other hand, different parameters are used to predict different categories of core performance indicators, so that the prediction results 


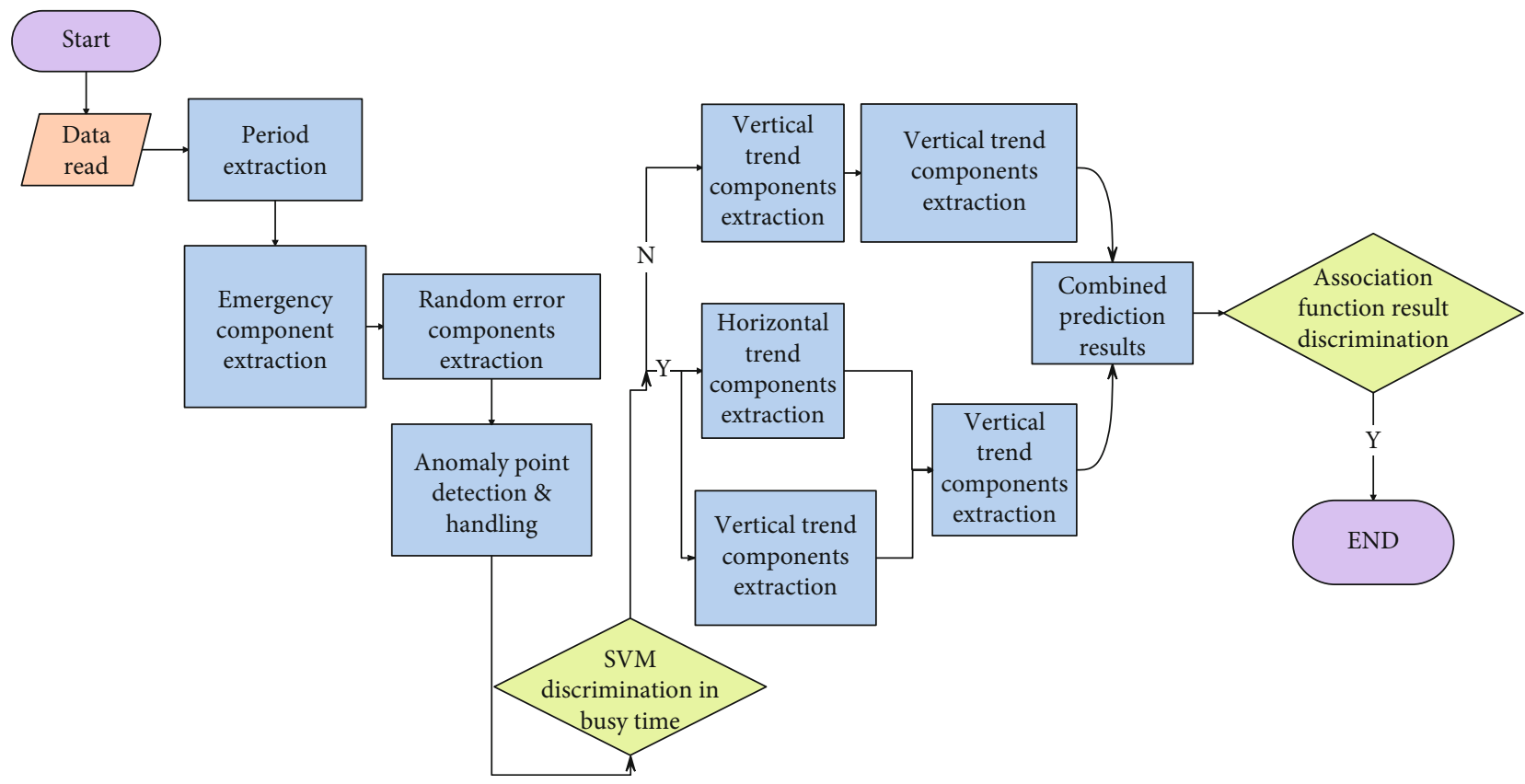

FIgURE 9: Flow chart of an improved big data prediction model based on SVM and association rules.

have higher accuracy. In time series, the series can be divided into stationary time series and nonstationary time series. A stationary series means that the mean and variance of the series have no systematic changes and strictly eliminate periodic changes. Intuitively speaking, the series have small fluctuations and no trend changes. The nonstationary time series corresponds to the stationary time series, and it is intuitively manifested as the series changes and fluctuates greatly. In the same time series, there are also stationary and nonstationary segments. For the core performance indicators of mobile communication networks in the form of time series, the numerical changes are also divided into stationary time series and nonstationary time series. For the core performance indicators of the mobile communication network, the stationary segment is called the nonbusy hour, and the nonstationary segment is called the busy hour. The so-called busy hour means that the core performance index of the cell fluctuates greatly over time, and the so-called nonbusy hour means that the change of the core performance index of the cell tends to be stable. In mobile communication networks, busy hours and nonbusy hours generally appear in day and night, weekends, and normal hours. Excessive cell network load is the main reason for busy hours, and the main goal of network optimization is to optimize network resources and reduce network load. Therefore, in the prediction of core performance indicators of mobile communication networks, busy hour performance indicators are more important. Figure 10 shows the busy and nonbusy hours of the RRC setting success rate in one cycle. Among them, the blue dot represents the value of the RRC setting success rate, the red area is the busy period, the green area is the nonbusy period, and the black line is the line where the dividing point between busy and nonbusy hours is located.

Since the predictive analysis of busy hour performance indicators is essential for network optimization, and the cor-

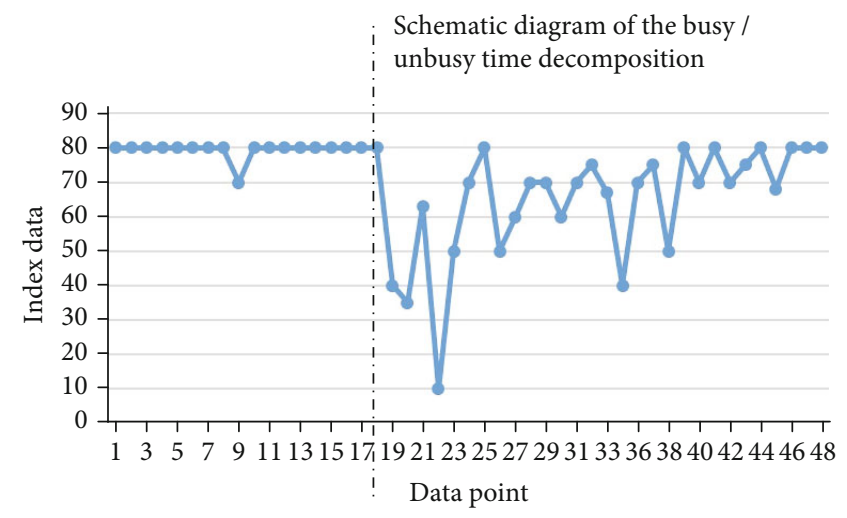

FIGURE 10: RRC setting success rate during busy/nonbusy time in a single cycle.

rect distinction between busy hour and nonbusy hour is the prerequisite guarantee for busy hour prediction, the traditional method of identifying busy hour and nonbusy hour is to find a demarcation point. The algorithm performs differential processing on points in a single cycle and takes the absolute value to obtain L-1 differential values and defines the minimum busy/nonbusy time length $N$ and the busy/nonbusy time threshold $x x$. The algorithm sequentially calculates the probability $P 1$ that the value of the first $N$ consecutive points is greater than the threshold value for point $i$ (range $N+1 \sim L$ ) and the probability $P 2$ that the value of the next consecutive $N$ points is less than the threshold value. Moreover, the algorithm calculates the comprehensive proportion $P 1+P 2$ or $2-P 1-P 2$ and selects the point with the maximum comprehensive proportion among all the points as the busy/nonbusy time boundary point. The length $N$ is user-defined and is at least one-third of the period value. If it is less than one-third of the period value, there 


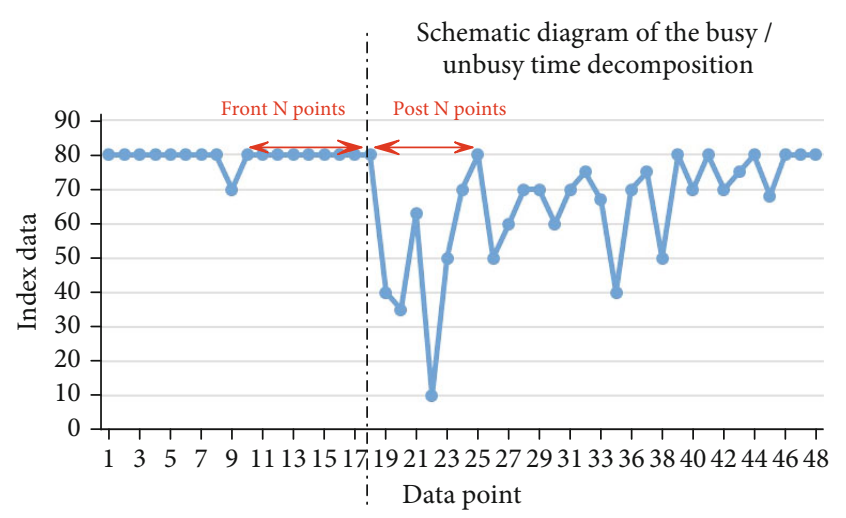

FIGURE 11: Discrimination rules for single-cycle busy/nonbusy time.

TABLE 1: The prediction effect of the intelligent prediction analysis system based on the embedded wireless communication network.

\begin{tabular}{lccccc}
\hline No. & $\begin{array}{c}\text { The method } \\
\text { of this paper }\end{array}$ & $\begin{array}{c}\text { The } \\
\text { method of } \\
{[18]}\end{array}$ & No. & $\begin{array}{c}\text { The method } \\
\text { of this paper }\end{array}$ & $\begin{array}{c}\text { The } \\
\text { method of } \\
{[18]}\end{array}$ \\
\hline 1 & 88.24 & 76.24 & 22 & 93.76 & 81.21 \\
2 & 94.80 & 87.79 & 23 & 89.75 & 80.83 \\
3 & 92.19 & 80.67 & 24 & 88.93 & 83.60 \\
4 & 95.61 & 86.90 & 25 & 93.63 & 81.24 \\
5 & 90.79 & 85.85 & 26 & 93.72 & 82.40 \\
6 & 90.34 & 80.82 & 27 & 86.57 & 81.64 \\
7 & 92.03 & 84.27 & 28 & 87.84 & 82.19 \\
8 & 89.99 & 79.78 & 29 & 88.02 & 80.66 \\
9 & 90.31 & 81.16 & 30 & 94.71 & 83.80 \\
10 & 92.04 & 84.58 & 31 & 91.93 & 79.36 \\
11 & 92.09 & 82.65 & 32 & 89.85 & 77.48 \\
12 & 91.54 & 84.08 & 33 & 88.75 & 82.56 \\
13 & 95.55 & 82.80 & 34 & 94.19 & 82.16 \\
14 & 88.22 & 83.24 & 35 & 86.38 & 75.30 \\
15 & 90.36 & 83.11 & 36 & 95.21 & 81.00 \\
16 & 89.64 & 81.32 & 37 & 86.80 & 76.10 \\
17 & 89.29 & 81.96 & 38 & 86.88 & 80.43 \\
18 & 91.78 & 81.04 & 39 & 94.31 & 80.29 \\
19 & 86.25 & 76.18 & 40 & 92.11 & 86.84 \\
20 & 94.62 & 84.86 & 41 & 92.51 & 78.70 \\
21 & 87.94 & 75.12 & 42 & 88.71 & 77.93 \\
\hline & & & & & \\
\hline
\end{tabular}

may be multiple busy/nonbusy demarcation points in a single period. The threshold is determined according to the fluctuation range of the performance index, and the general range is $0.1 \sim 1$. The figure below shows the rules for distinguishing between busy and nonbusy hours. The discriminant rule of single-cycle busy/nonbusy time is shown in Figure 11.

The method in this paper is compared with the literature 19 , and the effect of model prediction is comprehensively evaluated. The performance evaluation of the intelligent predictive analysis system based on the embedded wireless communication network is carried out, and its intelligent predictive effect and intelligent decision-making effect are
TABLE 2: Decision-making effect of intelligent predictive analysis system based on embedded wireless communication network.

\begin{tabular}{lccccc}
\hline No. & $\begin{array}{c}\text { The method } \\
\text { of this paper }\end{array}$ & $\begin{array}{c}\text { The } \\
\text { method of } \\
{[18]}\end{array}$ & No. & $\begin{array}{c}\text { The method } \\
\text { of this paper }\end{array}$ & $\begin{array}{c}\text { The } \\
\text { method of } \\
{[18]}\end{array}$ \\
\hline 1 & 85.72 & 74.78 & 22 & 83.25 & 76.66 \\
2 & 81.36 & 73.41 & 23 & 92.42 & 80.10 \\
3 & 88.89 & 78.69 & 24 & 81.01 & 70.97 \\
4 & 80.13 & 70.80 & 25 & 87.11 & 75.45 \\
5 & 92.68 & 84.75 & 26 & 87.70 & 74.73 \\
6 & 85.44 & 78.03 & 27 & 82.96 & 77.31 \\
7 & 79.71 & 72.31 & 28 & 84.74 & 76.48 \\
8 & 80.15 & 70.75 & 29 & 91.16 & 85.02 \\
9 & 88.64 & 81.93 & 30 & 92.08 & 79.88 \\
10 & 89.11 & 76.71 & 31 & 83.05 & 71.75 \\
11 & 80.95 & 76.68 & 32 & 90.59 & 79.47 \\
12 & 87.04 & 79.12 & 33 & 82.88 & 71.64 \\
13 & 89.74 & 77.02 & 34 & 80.17 & 71.58 \\
14 & 81.35 & 75.06 & 35 & 89.89 & 79.05 \\
15 & 84.31 & 79.19 & 36 & 81.59 & 71.31 \\
16 & 87.58 & 75.12 & 37 & 82.62 & 73.66 \\
17 & 79.58 & 70.11 & 38 & 84.82 & 72.23 \\
18 & 92.72 & 86.95 & 39 & 88.01 & 75.30 \\
19 & 87.48 & 77.94 & 40 & 86.60 & 82.19 \\
20 & 90.91 & 81.84 & 41 & 89.08 & 77.69 \\
21 & 80.71 & 76.12 & 42 & 83.85 & 71.71 \\
\hline & & & & & \\
\hline
\end{tabular}

counted, and the test results shown in Tables 1 and 2 below are obtained.

From the above research, it can be seen that the intelligent predictive analysis system based on the embedded wireless communication network proposed in this paper is very effective and has a positive effect on the construction and development of the embedded wireless communication network.

\section{Conclusion}

In order to further improve the network performance, it is necessary to study the network traffic and extract the parameters that can characterize the network traffic, so as to pass the modeling and performance analysis of the network traffic. At the same time, it is necessary to find adjustable performance parameters and implement effective control of traffic, thereby improving and optimizing network performance. In addition, today's networks are beginning to carry more and more application services, the scale of networks is getting larger and larger, and the characteristics of network behaviors are becoming more and more complex. This has brought huge challenges to network service quality, flow control, and network management planning, and contradictions have become increasingly prominent. This article takes the embedded wireless communication network as the research object, constructs the intelligent predictive analysis system, and validates and analyzes its performance. The 
research results show that the intelligent predictive analysis system based on the embedded wireless communication network proposed in this paper is very effective and has a positive effect on the construction and development of the embedded wireless communication network.

The research work in this paper is mainly to carry out distributed predictive control research for the agent of the nonlinear nominal model, but the influence of uncertainty on the system is not fully considered, such as the uncertainty of the agent model, the uncertainty of external interference, and communication uncertainty between agents. In view of these problems, the traditional robust predictive control method can be used for reference, but it cannot be simply and directly extend to distributed situations, so the followup further research needs to focus on the above problems and performance improvement.

Because the forecasting process is affected by many factors, the regression-based forecasting method has complex characteristics, which makes its forecasting have its complex characteristics. At the same time, each forecasting model has its own shortcomings and adaptability. Therefore, as far as the current research methods and prediction models are concerned, a lot of research is still needed, which is also the next step.

\section{Data Availability}

The labeled dataset used to support the findings of this study are available from the corresponding author upon request.

\section{Conflicts of Interest}

The author declares no competing interests.

\section{Acknowledgments}

This study is sponsored by Hefei University of Technology.

\section{References}

[1] A. Al-Halafi and B. Shihada, "UHD video transmission over bidirectional underwater wireless optical communication," IEEE Photonics Journal, vol. 10, no. 2, pp. 1-14, 2018.

[2] J. Barowski, M. Zimmermanns, and I. Rolfes, "Millimeterwave characterization of dielectric materials using calibrated FMCW transceivers," IEEE Transactions on Microwave Theory and Techniques, vol. 66, no. 8, pp. 3683-3689, 2018.

[3] B. Behroozpour, P. A. M. Sandborn, M. C. Wu, and B. E. Boser, "Lidar system architectures and circuits," IEEE Communications Magazine, vol. 55, no. 10, pp. 135-142, 2017.

[4] Q. Y. Cheng, X. L. Zhao, Y. X. Weng, Y. D. Li, and J. B. Zeng, "Fully sustainable, nanoparticle-free, fluorine-free, and robust superhydrophobic cotton fabric fabricated via an eco-friendly method for efficient oil/water separation," ACS Sustainable Chemistry \& Engineering, vol. 7, no. 18, pp. 15696-15705, 2019.

[5] Y. Jiang, S. Karpf, and B. Jalali, "Time-stretch LiDAR as a spectrally scanned time-of-flight ranging camera," Nature Photonics, vol. 14, no. 1, pp. 14-18, 2020.

[6] H. C. Kumawat and A. B. Raj, "Extraction of Doppler signature of micro-to-macro rotations/motions using continuous wave radar-assisted measurement system," IET Science, Measurement \& Technology, vol. 14, no. 7, pp. 772-785, 2020.

[7] Y. J. Ma, A. Tadros, J. du, and E. Y. Chang, "Quantitative twodimensional ultrashort echo time magnetization transfer (2D UTE-MT) imaging of cortical bone," Magnetic Resonance in Medicine, vol. 79, no. 4, pp. 1941-1949, 2018.

[8] N. Maring, P. Farrera, K. Kutluer, M. Mazzera, G. Heinze, and H. de Riedmatten, "Photonic quantum state transfer between a cold atomic gas and a crystal," Nature, vol. 551, no. 7681, pp. 485-488, 2017.

[9] Z. Meng, J. Li, C. Yin et al., "Dual-band dechirping LFMCW radar receiver with high image rejection using microwave photonic I/Q mixer," Optics Express, vol. 25, no. 18, pp. 2205522065, 2017.

[10] H. Mohapatra and A. K. Rath, "Detection and avoidance of water loss through municipality taps in India by using smart taps and ICT," IET Wireless Sensor Systems, vol. 9, no. 6, pp. 447-457, 2019.

[11] J. Pan, Q. Xie, H. Chiang et al., "“From the nature for the nature": an eco-friendly antifouling coating consisting of poly(lactic acid)-based polyurethane and natural antifoulant," ACS Sustainable Chemistry \& Engineering, vol. 8, no. 3, pp. 1671-1678, 2020.

[12] Z. Sabouri, A. Akbari, H. A. Hosseini, A. Hashemzadeh, and M. Darroudi, "Eco-friendly biosynthesis of nickel oxide nanoparticles mediated by okra plant extract and investigation of their photocatalytic, magnetic, cytotoxicity, and antibacterial properties," Journal of Cluster Science, vol. 30, no. 6, pp. 1425-1434, 2019.

[13] A. Seri, G. Corrielli, D. Lago-Rivera et al., "Laser-written integrated platform for quantum storage of heralded single photons," Optica, vol. 5, no. 8, pp. 934-941, 2018.

[14] S. Sharaf and M. E. El-Naggar, "Eco-friendly technology for preparation, characterization and promotion of honey bee propolis extract loaded cellulose acetate nanofibers in medical domains," Cellulose, vol. 25, no. 9, pp. 5195-5204, 2018.

[15] K. Soga and L. Luo, "Distributed fiber optics sensors for civil engineering infrastructure sensing," Journal of Structural Integrity and Maintenance, vol. 3, no. 1, pp. 1-21, 2018.

[16] L. J. Xu, X. Lin, Q. He, M. Worku, and B. Ma, "Highly efficient eco-friendly X-ray scintillators based on an organic manganese halide," Nature Communications, vol. 11, no. 1, pp. 1-7, 2020.

[17] T. Zhong, J. M. Kindem, J. G. Bartholomew et al., "Nanophotonic rare-earth quantum memory with optically controlled retrieval," Science, vol. 357, no. 6358, pp. 1392-1395, 2017.

[18] G. Aceto, G. Bovenzi, D. Ciuonzo, A. Montieri, V. Persico, and A. Pescapé, "Characterization and prediction of mobile-app traffic using markov modeling," IEEE Transactions on Network and Service Management, vol. 18, no. 1, pp. 907-925, 2021.

[19] A. Montieri, G. Bovenzi, G. Aceto, D. Ciuonzo, V. Persico, and A. Pescapè, "Packet-level prediction of mobile-app traffic using multitask deep learning," Computer Networks, vol. 200, article 108529, 2021.

[20] F. Zhang, Q. Guo, and S. Pan, "Photonics-based real-time ultra-high-range-resolution radar with broadband signal generation and processing," Scientific Reports, vol. 7, no. 1, pp. 1-8, 2017.

[21] T. Zhong and P. Goldner, "Emerging rare-earth doped material platforms for quantum nanophotonics," Nano, vol. 8, no. 11, pp. 2003-2015, 2019. 